\title{
OBESIDAD Y SOBREPESO EN ESTUDIANTES DEL NIVEL PRIMARIO DEL COLEGIO CRISTO REY - TACNA 2007
}

\section{RESUMEN}

La obesidad infantil ha aumentado en el mundo, y con ello sus riesgos a largo plazo. Objetivo: Determinar la prevalencia de obesidad y sobrepeso en niños prépuberes en el Colegio Cristo Rey de Tacna. Material y Métodos: fueron seleccionados al azar 218 alumnos del nivel primario , se obtuvo el peso, talla y edad y se determinó el índice de masa corporal. Resultado: Se encontró una prevalencia global de $26 \%$ de sobrepeso y $39 \%$ de obesidad.

Conclusión: El alto consumo de energía y el poco gasto calórico demostrado por la poca actividad física son factores predisponentes para desarrollar obesidad y sobrepeso.

\begin{abstract}
The prevalence of obesity in children is having a progressive increase in the World. Objective: to determine the prevalence of obesity in 6 to 10 years old pre puberal scholars from Cristo Rey - Tacna. Material and Methods: We selected randomized 218 students, 6 to 10 years old pre puberal children from Cristo Rey school, Tacna. Age, weight and height was recorded, and BMI was calculated. Results: The prevalence of overweight was $26 \%$ and obesity was $39 \%$. Conclusion: We concluded a high prevalence of overweight and obesity in pre puberal children and reinforces the necessity of education in healthy habits of nutrition and activity to prevent the development of abnormal weight during the school years.
\end{abstract}

(Key words: obesity, overweight)

\section{INTRODUCCION}

La obesidad o exceso de grasa en el cuerpo de los niños es causa de preocupación, ya que aumenta el riesgo de enfermedades durante la infancia y la edad adulta. Los niños que son obesos tienen la presión más alta, problemas ortopédicos de rodillas, propensión alta a desarrollar diabetes tipo 2 y problemas psicosociales, debido al rechazo de la sociedad a individuos obesos.

Los factores que contribuyen a la aparición de obesidad en los niños son variados, entre los cuales se puede citar la herencia, el sedentarismo, un excesivo consumo de alimentos y hábitos alimentarios inadecuados.

El aumento excesivo del peso corporal en los últimos 30 años está asociado a cambios en los hábitos de ingesta y de actividad física. El desarrollo industrial, la mejoría del poder adquisitivo, los cambios socioeconómicos y los avances tecnológicos se cuentan entre los responsables.

En nuestro país existen pocos estudios sobre la obesidad y sobrepeso en estudiantes. Es por esto importante comprender las causas de las tendencias recientes en el aumento de la obesidad en estudiantes del nivel primario.
El objetivo del presente estudio fue determinar la prevalencia de obesidad y sobrepeso en los alumnos de nivel primario del Colegio Cristo Rey y algunas de las causas de su aparición. Asimismo, se llevaron a cabo dos encuestas para conocer los hábitos alimenticios y para determinar el nivel de actividad física.

\section{Definición de Términos:}

- Índice de Masa Corporal (imc): relación del peso expresado en kilogramos, sobre el cuadrado de la talla expresada en metros.

- Obesidad: Niños con IMC mayor al percentil 95.

- Sobrepeso: Niños con IMC mayor al percentil 85 , hasta el percentil 95.

- Normal: Niños con IMC entre percentiles 10 y85.

- Bajopeso: Niños con IMC menor al percentil 10.

- Prevalencia: La proporción (expresada en porcentaje) de individuos de un grupo o una población que presentan una característica o evento determinado en un momento o periodo de tiempo determinado. 


\section{MATERIALES YMÉTODOS}

Población de estudio: Está formada por 434 alumnos de edades comprendidas entre los $5 y$ 11 años, pertenecientes al nivel primario $\left(1^{\circ}\right.$ a $6^{\circ}$ grado) del Colegio Cristo Rey.

Muestra: Con el fin de obtener una muestra representativa se consideró una confianza de $95 \%$ y un error máximo aceptable de exactitud probabilística de $5 \%$. Se introdujeron los datos en el programa STATS (Hernández. Metodologia de la Investigación. Mc Graw Hill, 3a. edición. México) y de la población de 434 se obtuvo una muestra de 218 alumnos.

Fuentes de Información: Se obtuvieron los datos de talla, peso y edad cronológica de la enfermería del Colegio. Se llevaron a cabo también, dos encuestas para conocer los hábitos alimenticios y determinar el nivel de actividad física de los alumnos.

Luego se aplicó la fórmula para determinar el Índice de Masa corporal (IMC): Peso dividido por el cuadrado de la talla. Estos valores obtenidos fueron llevados a la tabla "2 a 20 años: Niños Percentiles del Indice de Masa Corporal por Edad" para obtener el percentil de cada uno de los alumnos, y poder así clasificarlos dentro de alguna de las categorias: obesidad, sobrepeso, normal o bajo peso. Asimismo, se llevaron a cabo dos encuestas, una para conocer los hábitos alimenticios y otra para determinar el nivel de actividad física de los alumnos.

Resultado: Fueron evaluados un total de 218 alumnos del nivel primario, del $1^{\circ}$ al $6^{\circ}$ año.

Fueron evaluados un total de 218 alumnos del nivel primario, del $1^{\circ}$ al $6^{\circ}$ año.

Se encontró una prevalencia global de $26 \%$ de sobrepeso y $39 \%$ de obesidad.

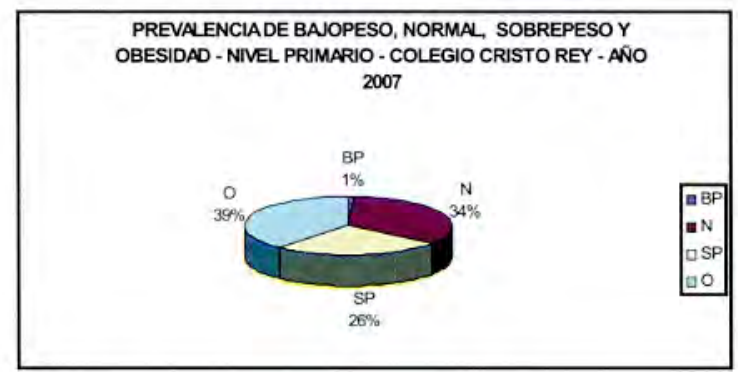

En relación con la pregunta si practicaban algún deporte además de la actividad física realizad en el curso de educación física, 39\% respondió que no practicaba ningún deporte, $34 \%$ practicaba fútbol, $11 \%$ practicaba basket, $7 \%$ lo hacia con karate.

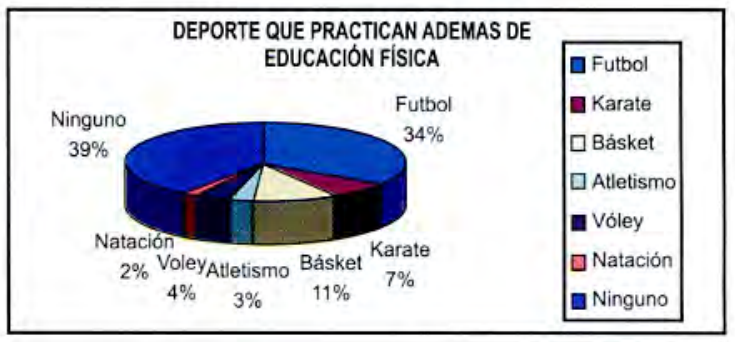

Con relación al tiempo que practican deporte semanalmente, $26 \%$ respondió que lo hacia menos de 1 hora; $33 \%$ lo realizaba por una hora en promedio y $41 \%$ lo practicaba por más de una hora.

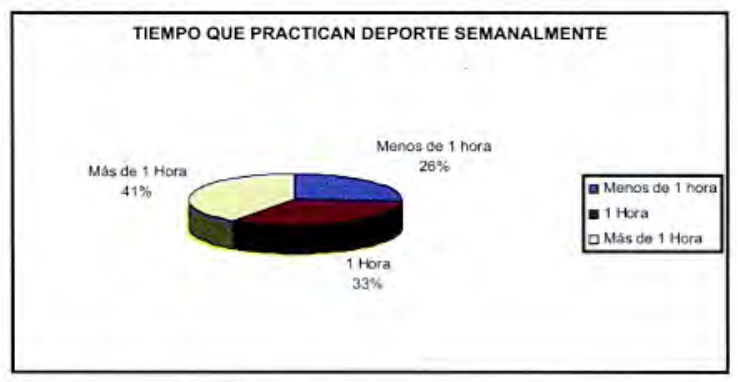

Respecto al tiempo dedicado a ver televisión durante la semana, $21 \%$ respondió que lo hacía por menos de una hora, $31 \%$ respondió que lo hacía durante una hora y $31 \%$ respondió que lo hacia por más de una hora.

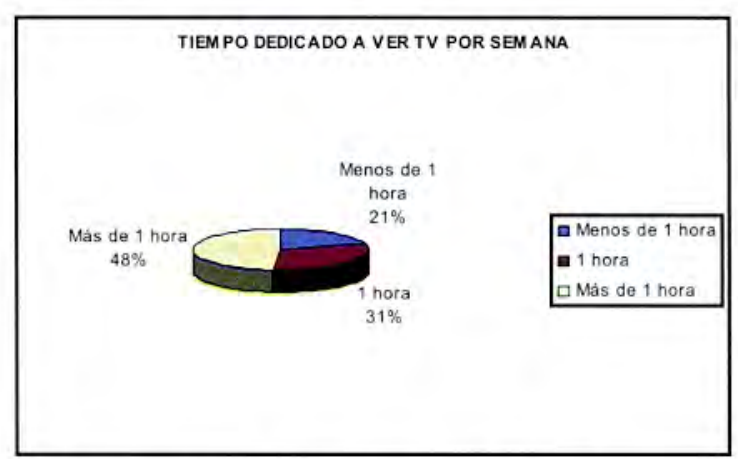

En relación al tiempo dedicado a la computadora y videojuegos semanalmente $31 \%$ respondió que lo hacía menos de 1 hora, $41 \%$ lo hacia durante 1 hora y $28 \%$ lo hacía por más de 1 hora.

TIEMPO QUE DEDICAN A VIDEOJUEGOS O COMPUTADORA POR SEMANA

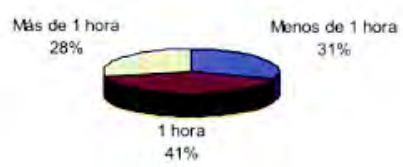

a Menos de 1 hora

E 1 hora

a Mas de 1 hora 
Con respecto al consumo de alimentos en el colegio, además del que llevan en la lonchera, $62 \%$ respondió que sí consumian algo más siendo galletas, empanadas y snacks en su mayoría, como se aprecia en el cuadro adjunto.

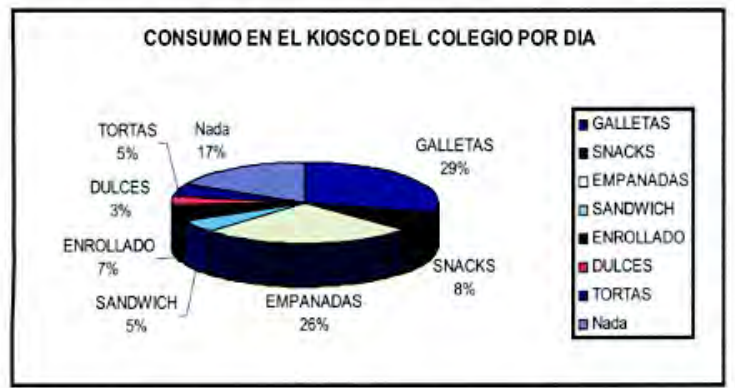

Discusión: La obesidad ha adquirido las características de ser un gran problema en los niños, no sólo en los países desarrollados sino también en los de América Latina. Se ha demostrado que los niños que son obesos tienen una alta probabilidad de continuar siendo obesos en la edad adulta y desarrollar enfermedades crónicas, como las cardiovasculares y la diabetes.

Las cifras halladas nos muestran una alta prevalencia de obesidad y sobrepeso en los niños del nivel primario del Colegio Cristo Rey.

Los datos obtenidos en nuestro trabajo de sobrepeso $(26 \%)$ y obesidad $(39 \%)$ son muy superiores a los observados en un estudio realizado en niños de colegios nacionales en el distrito de San Martín de Porras de Lima, donde la prevalencia de sobrepeso era $9,96 \%$ y de obesidad $5,62 \%$.

También se puede decir que están muy por encima de lo hallado en un estudio realizado en Chile en La Clínica Las Condes (perteneciente a un estrato socioeconómico medio alto), en la cual hallaron un sobrepeso de $16,1 \%$ y de obesidad de $15,8 \%$.

Asimismo, de los datos de la encuesta podemos deducir que un gran porcentaje de niños (39\%) no practica ningún deporte, lo que los convierte en grandes sedentarios.

Por otro lado, a la no práctica del deporte se suma el hecho de son llevados al colegio en vehículos desde la puerta de su casa, lo que no les permite hacer algún ejercicio adicional.

Asimismo, la gran mayoría de los alumnos pasan más de 1 hora frente al televisor, la computadora y los videojuegos, lo que inciden en la poca actividad física desarrollada por ellos.

\section{CONCLUSIONES}

1.La obesidad infantil encontrada en el nivel primario del Colegio Cristo Rey tiene una prevalencia de $39 \%$.

2.Existe una prevalencia de sobrepeso de $26 \%$ en los niños del nivel primario.

3.Los factores más relacionados con la obesidad y sobre peso son el SEDENTARISMO, poca actividad física, tiempo dedicado frente al televisor, computadora y/o videojuegos.

4.Existe una gran tendencia al consumo de alimentos de alto contenido calórico y también a ingerir golosinas, bebidas gaseosas, snacks, los cuales son alimentos ricos en hidratos de carbono.

5.El alto consumo de energía y el poco gasto calórico demostrado por la poca actividad física son factores predisponentes para desarrollar obesidad.

\section{RECOMENDACIONES}

Se hace necesario tomar conciencia de que la obesidad es una enfermedad prevenible cuya presencia tiene serias consecuencias para la salud de la población afectada. Sería necesario implementar programas preventivos, como charlas nutricionales e incrementar la actividad física.

\section{BIBLIOGRAFIA}

Http://es.wikipedia.org/wiki/Prevalencia; Prevalencia.

Hernández, Roberto: Metodología de la Investigación Cientifica, 299-340. Editorial Mc Graw Hill, 3a edición. México, 2003.

Pisabarro, Raúl y Col.: ENSO niños 1: "Primera encuesta Nacional de sobrepeso y obesidad en niños uruguayos". Revista Médica Uruguay 2002; 18:244-250. Laboratorios Roche. Montevideo.

Santos Muñoz, S : "La Educación Física escolar ante el problema de la Obesidad y el Sobrepeso". Revista Internacional de la Medicina y Ciencias de la Actividad Fisica y el Deporte. Número 19. Año 2005, 355-360. Edit Ibérica. Madrid.

Vásquez, C: "La grasa como factor de riesgo de obesidad en la población infantil". Revista de Endocrinología y Nutrición, 2003; 50(6): 198209. Madrid. 
Who. Diet, Nutrition and the prevention of chronic diseases. World health Organization/FAO experts consultation. Genova; 2002.

www.cdc.gov/growthcharts/. 2 a 20 años: Niños. Percentiles del Índice de Masa Corporal por edad. Centro Nacional de Estadísticas de Salud en colaboración con el Centro Nacional para la Prevención de Enfermedades Crónicas y Promoción de Salud (2000).

www.nutrinfo.com.ar; PINO.CHO, Proyecto de Investigación de la Niñez y la Obesidad. Dr. Nelio Bazán.

www.scielo.org.pe/; Distribución del Índice de masa corporal (IMC) y prevalencia de obesidad primaria en niños pre-púberes de 6 a 10 años de edad en el distrito de San Martín de Porras, Lima.

Revista Médica Herediana, 14 n3 -2003.

www.scielo.cl/: Prevalencia de sobrepeso y obesidad en niños que se controlan en pediatria ambulatoria en Clínica las Condes.

Eyzaguirre, Francisca y col. Revista Chilena de Pediatria 76(2);143-149, 2005.

www.nutrar.com.ar/; Estudio Prevalencia de Obesidad en escolares de 7 a 9 años de edad de niveles sociales extremos. Centro Nacional de Investigaciones Nutricionales. 